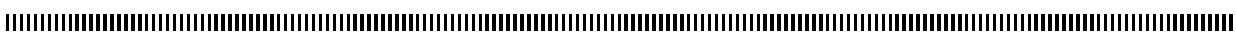

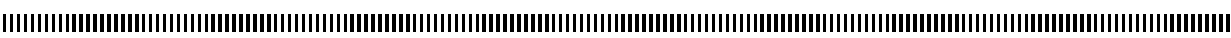

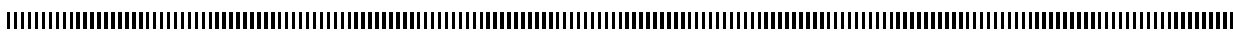

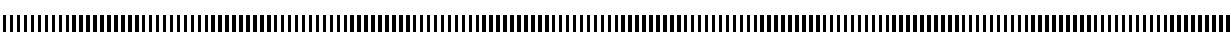

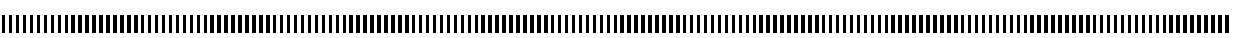
|

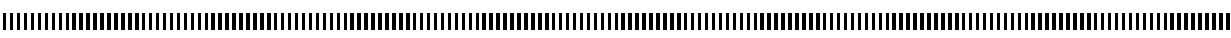

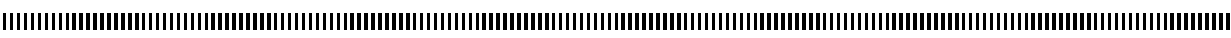

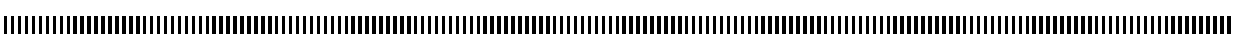
|

\title{
Analysis of a model describing stage-structured population dynamics using hawk-dove tactics
}

\author{
Ali Moussaoui ${ }^{1,2, *}$, Nguyen Ngoc Doanh ${ }^{3}$, Pierre Auger $^{4}$ \\ ${ }^{1}$ Department of Mathematics. Faculty of sciences. University of Tlemcen, Algeria \\ ${ }^{2}$ Applied Mathematics Laboratory of Le Havre, Normandy Mathematics Federation (FR CNRS \\ 3335).moussaoui.ali@gmail.com \\ ${ }^{3}$ School of Applied Mathematics and Informatics, Hanoi University of Sciences and Technology, \\ No 1 Dai Co Viet, Hai Ba Trung District, Hanoi, Vietnam. doanhbondy@gmail.com \\ ${ }^{4}$ UMI IRD 209, UMMISCO, IRD France Nord, 32 ave. Henri Varagnat, F-93143, Bondy, France. \\ pierre.auger@ird.fr
}

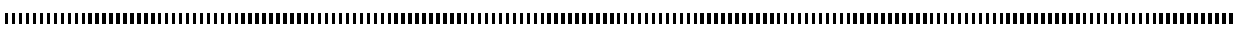

ABSTRACT. The purpose of this paper is to investigate the effects of conflicting tactics of resource acquisition on stage structured population dynamics. We present a population subdivided into two distinct stages (immature and mature). We assume that immature individual survival is densitydependent. We also assume that mature individuals acquire resources required to survive and reproduce by using two contrasted behavioral tactics (hawk versus dove). Mature individual survival thus is assumed to depend on the average cost of fights while individual fecundity depends on the average gain in the competition to access the resource. Our model includes two parts: a fast part that describes the encounters and fights involves a game dynamic model based upon the replicator equations, and a slow part that describes the long-term effects of conflicting tactics on the population dynamics. The existence of two time scales let us investigate the complete system from a reduced one, which describes the dynamics of the total immature and mature densities at the slow time scale. Our analysis shows that an increase in resource value may decrease total population density, because it promotes individual (i.e. selfish) behavior. Our results may therefore find practical implications in animal conservation or biological control for instance.

RÉSUMÉ. Le but de cet article est d'étudier les effets de comportements agressifs dans l'acquisition de ressources sur la dynamique d'une population structurée en deux classes d'âge. Nous considérons une population divisée en deux sous populations distinctes (immatures et adultes matures). Nous supposons que la survie individuelle de la population immature est densité dépendante. Nous supposons également que les individus matures sont en compétition pour acquérir les ressources nécessaires pour leur survie et leur reproduction. Les adultes utilisent deux tactiques comportementales (faucon et colombe). Lors de confrontations entre adultes, la survie d'un individu mature est supposée être affectée par le coût moyen des combats alors que la fécondité dépend du gain moyen obtenu en accédant à la ressource. Notre modèle comprend deux parties : une partie rapide qui décrit les rencontres et les combats basée sur les équations du réplicateur, et une partie lente qui décrit les effets à long terme des tactiques conflictuelles sur la dynamique de la population. L'existence de deux échelles de temps nous permet d'étudier le système complet à partir d'un système réduit, qui 
128 - ARIMA - Volume 20 - 2015

décrit la dynamique des densités totales des immatures et des adultes à l'échelle de temps lente. Notre analyse montre que le taux de croissance global de la population dépend de la valeur de la ressource et du coût des combats entre adultes. Nos résultats trouvent des implications pratiques dans la conservation des espèces et le contrôle biologique.

KEYWORDS : Hawk and dove game, aggregation of variables, population dynamics.

MOTS-CLÉS : Jeux faucon-colombe, agrégation des variables, dynamique des populations.

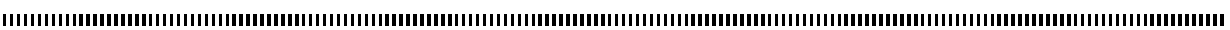


This article is an extended version of an article published in the conference proceedings CARI 2012 (Moussaoui and al. 2012). Actually, it contains no new results in relation to the initial publication. Nevertheless, it has many new elements such as a more extensive bibliography, a detailed mathematical analysis of a planar system and a substantial improvement in the presentation of the study.

\section{Introduction}

It is the fact that individuals are the building blocks of ecological systems. The properties and behavior of individuals determine the properties of the systems they compose. The challenging question is "how can we explain the effects of individuals' behaviors on the system by using models?" There were some investigations that try to answer this question by studying the effects of the variation in individual behavior on population dynamics and stability of communities (Lott, 1991; Davies and Krebs, 1993; Manning and Dawkins, 1998; Sutherland and Norris, 2002). The ability for an individual to survive and reproduce depends on its behavior (Davies and Krebs, 1993). In the case of competition, one of the major processes that govern the evolution of ecological systems, where individuals compete for resources, we can expect that individuals will develop behavioral tactics according to their phenotypic quality in order to maximize food acquisition (Manning and Dawkins, 1998). This led to the concept of so called behavior-based models (Sutherland and Norris, 2002).

Recently, some authors presented an approach aimed at linking individual variation in behavior and population dynamics. For instance, in Auger et al. 2002, 2006 and Auger and Pontier 1998, mathematical models are developed in order to couple a game model that describes interactions among predator individuals (Maynard Smith, 1982; Sigmund, 1986; Hofbauer and Sigmund, 1998; Cressman, 1992, 1995; Mesterton-Gibbons, 1992; ) with a predator-prey model; in Auger et al.,1998; Auger et al. 2001 and Moussaoui et al., 2014, game dynamics are coupled with competition dynamics. In these studies, the authors look for the effects of individuals behavior (hawks and doves) on the dynamics of the whole systems. We also refer to (Chambon-Dubreuil et al. 2006, Marva et al. 2013) where the authors studied a discrete age-structured population model linking a game model that describes interactions among adult individuals with a model that describes population growth (a Leslie matrix). In that case, a classical Leslie matrix model is used to describe the population demography. Adults acquire resources required to survive and reproduce by using two contrasted behavioral tactics ( hawk versus dove). Individual survival depends on the cost of flights while individuals fecundity depends on gain in the competition to access the resource. The authors analyzed the effects of conflicting tactics of resource acquisition on population dynamics.

In this present contribution, we are also interested in this linking approach. Contrary to previous cases in Chambon-Dubreuil et al. 2006 and Marva et al. 2013, using discrete time models, we consider in the present work a continuous stage structured model 
which incorporates individual behavior of the mature stage. Most classical stage structured models do not take into account the behavioral structure of the population. Here, we shall take into account different tactics that mature can use for exploiting a common resource. We consider a population with two stages: immature and mature. Immature individual survival is assumed to be density-dependent while mature individuals compete for a given resource that is required to survive and/or reproduce. To gain access to this resource, mature individuals display different behavioral tactics. We consider that individuals can use two different tactics: hawk $(H)$ or dove $(D)$ (Hofbauer and Sigmund, 1998; Maynard Smith, 1982). The hawk is always aggressive and fights in any case. The dove is never aggressive and never escalates. When a hawk encounters a dove, it is always the winner and obtains a reward $G$, which represents an access to a resource. When two hawks encounter, they fight. One of them is the winner. However, both of them can receive injuries due to fighting. When two doves meet, they never fight and in average they share the gain. We assume that each mature individual can use both tactics along its life. In this way, the same individual is not always hawk or dove but it can change tactics. Whenever an encounter occurs, a mature individual could use the hawk tactic, but in some other occasions, it could use the dove tactic. After a certain number of encounters, we assume that, on the average, mature individuals are using the tactic that gives them the best payoff. To describe the change of tactics, we are going to use a game dynamic model based upon the replicator equations (Hofbauer and Sigmund,1998, Cressman.,1992, 1995; Mesterton-Gibbons, 1992).

We also assume two different time scales:

- A fast time scale corresponds to the disputes and fights between the mature individual's that occur each day.

- A slow time scale corresponds to the growth of population, the mortality and the birth of immature.

Fecundity and survival usually depend on resource availability (Begon et al., 1996). The abundance of the resource is expected to modify the intensity of the competition among individuals. Therefore, we also included in our model the dependence of both fecundity and mortality on the average gain and cost of the game in the long term.

As two time scales are involved in the dynamics, we can use aggregation methods to build a reduced model (the aggregated model) that describes the dynamics of the two stage population at the slow-time scale (Iwasa et al., 1987 and 1989; Auger and Bravo de la Parra, 2000; Auger et al. 2008a and 2008b; Auger and Poggiale, 1998).

The aim of this work is to study the effects of mature individual behavior on the dynamics of the stage structured population system. In particular, we shall investigate the effect of gain and cost parameters on the total density of population. We are interested in the coupled dynamics of, on one hand, mature changes of individual tactics at the fast time scale and, on the other hand, changes of demography corresponding to reproduction and mortality at the slow time scale. 


\section{Presentation of the model}

We consider a population subdivided into two distinct stages: immature and mature. The immature sub-population do not participate to reproduction. The mature individuals are assume to compete to access resource required to survive and to reproduce. Mature reproductive individuals are split into two different tactics: aggressive (hawks) and nonaggressive (doves). Let $n_{1}(t)$ be the size of the immature individual and $n_{2}(t)$ the total size of the mature individuals population at time $t$. The model is composed of two parts, a fast part that describes the change of tactics of mature and a slow part that describes the demographic process. We are going to present these two parts by using two sub-models as follows.

\subsection{The hawk-dove sub-model at the fast time scale}

The mature population is divided into two sub-populations: hawk mature and dove mature. Let $n_{2}^{H}(t)$ and $n_{2}^{D}(t)$ be respectively the hawk and dove mature individuals densities at time $t$. The total density of mature is given by $n_{2}(t)=n_{2}^{H}(t)+n_{2}^{D}(t)$. Mature individuals are assumed to compete to access resources. This process occurs at the fast time scale.

We use the hawk-dove game in order to represent the interaction between two mature sub-populations. The winner of the game gains an access to a common resource. Therefore, we assume that the gain $G$ is identical for all individuals. $C$ is the cost of loosing an escalated fight. The payoff obtained by hawks and doves when interacting are represented by the classical payoff matrix $A$ (Hofbauer and Sigmund, 1998):

$$
A=\left(\begin{array}{cc}
\frac{G-C}{2} & G \\
0 & \frac{G}{2}
\end{array}\right)
$$

Let $x(t)$ and $y(t)$ be respectively the hawks and doves proportions of mature individuals at time $t$.

$$
x(t)=\frac{n_{2}^{H}(t)}{n_{2}(t)}, \quad y(t)=1-x(t)=\frac{n_{2}^{D}(t)}{n_{2}(t)} .
$$

We also use the replicator equations that describe the change of tactics of adult individuals that we now briefly recall. At time $t$, the gain $\Delta_{H}$ of an individual always using the hawk strategy against a population with a proportion $x(t)$ of hawks and $y(t)$ of doves is the following one:

$$
\Delta_{H}=(1,0) A\left(\begin{array}{l}
x \\
y
\end{array}\right)
$$

The gain $\Delta_{D}$ of an individual always playing the dove strategy is the following one:

$$
\Delta_{D}=(0,1) A\left(\begin{array}{l}
x \\
y
\end{array}\right)
$$


The average gain of an individual playing the two tactics in proportions $(x(t), y(t))$ corresponding to the actual distribution of hawks and doves in the total population is the following one:

$$
\Delta=(x, y) A\left(\begin{array}{l}
x \\
y
\end{array}\right) .
$$

Let us calculate for each tactic, the difference between the gain of each of them and the average gain of the population. If this difference is positive (resp. negative), it is assumed that the proportion of players of this strategy is going to increase, (resp. decrease). With these assumptions, the replicator equations read

$$
\left\{\begin{array}{l}
\frac{d x}{d \tau}=x\left(\Delta_{H}-\Delta\right) \\
\frac{d y}{d \tau}=y\left(\Delta_{D}-\Delta\right)
\end{array}\right.
$$

Using this model, we assume that if a tactic brings a better payoff than the average payoff of the population, the proportion of individuals playing this strategy is increasing and conversely. In the previous equations, $\tau$ is the fast time scale. Thus, we assume that the hawk-dove game is fast in comparison to other processes that we shall now consider in the model, such as birth, death and the transformation from the immature individuals to mature individuals.

\subsection{A stage structured model at the slow time scale}

The assumptions of this model are as follows:

1. The immature population: the birth rate into the immature population is proportional to the existing mature population with a fecundity coefficient that depends on average gains $\bar{G}_{i}, i=H, D$; the death rate is proportional to the existing immature population with proportionality $\mu_{1}$. We also assume that the immature individual survival is densitydependent with the effect coefficient $-\eta$.

2. For the mature individuals: The transformation rate from the immature individuals to mature individuals is proportional to the existing immature population with proportionality $\beta$. For the mature equations, we assume that escalated contests cause injuries and therefore provoke a increase of the mortality of mature individuals according to the following relationship: $\mu_{2}\left(\bar{C}_{i}\right)=\mu+\alpha \bar{C}_{i}$, where $\mu$ is the constant natural mortality rate supposed identical for hawks and doves, $\bar{C}_{i}$ is the average cost, $i=H, D$ and $\alpha$ a positive coefficient which permits to regulate the effect of the average cost on the survival.

$$
\left\{\begin{array}{l}
\frac{d n_{1}}{d t}=F_{H}\left(\overline{G_{H}}\right) n_{2}^{H}+F_{D}\left(\overline{G_{D}}\right) n_{2}^{D}-\mu_{1} n_{1}-\beta n_{1}-\eta n_{1}^{2} \\
\frac{d n_{2}^{H}}{d t}=q \beta n_{1}-\mu_{2}\left(\overline{C_{H}}\right) n_{2}^{H} \\
\frac{d n_{2}^{D}}{d t}=(1-q) \beta n_{1}-\mu_{2}\left(\overline{C_{D}}\right) n_{2}^{D}
\end{array}\right.
$$


Parameter $q$ represents the proportion of immature individuals becoming hawks when surviving to mature state (respectively $(1-q)$ for doves).

The average cost is calculated by adding the cost of each type of encounter weighted by the proportion of this type of encounter. Therefore, the average cost received by a dove, that we note $\overline{C_{D}}$ is null, because doves do not fight and do not get injured:

$$
\overline{C_{D}}=0 \frac{n_{2}^{H}}{n_{2}}+0 \frac{n_{2}^{D}}{n_{2}}=0
$$

As a consequence, dove mortality $\mu_{2}\left(\overline{C_{D}}\right)$ is simply $\mu$.

$$
\mu_{2}\left(\overline{C_{D}}\right)=\mu
$$

On the contrary, hawks fight and get injured which causes them higher mortality risks than doves. Hawk mortality is a function of the average cost received by a hawk, that we note $\overline{C_{H}}$ :

$$
\overline{C_{H}}=\left(\frac{C}{2}\right) \frac{n_{2}^{H}}{n_{2}}+0 \frac{n_{2}^{D}}{n_{2}}=\left(\frac{C}{2}\right) \frac{n_{2}^{H}}{n_{2}}
$$

The hawk mortality $\mu_{2}\left(\overline{C_{H}}\right)$ is thus given by the following expression:

$$
\mu_{2}\left(\overline{C_{H}}\right)=\mu+\alpha\left(\frac{C}{2}\right) \frac{n_{2}^{H}}{n_{2}}
$$

\subsubsection{Fecundity}

For some species, the amount of resource has a continuous effect on fecundity, while for others, individuals must accumulate a given amount of resource before reproduction. Average gains are calculated by adding the gain of each type of encounter weighted by the proportion of this type of encounter. Thus, we obtain the next expressions for hawk $(H)$ and dove $(D)$ average gains:

$$
\begin{aligned}
\overline{G_{H}} & =\left(\frac{G}{2}\right) \frac{n_{2}^{H}}{n_{2}}+G \frac{n_{2}^{D}}{n_{2}} \\
\overline{G_{D}} & =0 \frac{n_{2}^{H}}{n_{2}}+\frac{G}{2} \frac{n_{2}^{D}}{n_{2}}=\left(\frac{G}{2}\right) \frac{n_{2}^{D}}{n_{2}}
\end{aligned}
$$

First, in order to study the effect of the resource on fecundity, we use a Holling type function. According to this function, fecundity increases with the average gain and then reaches a plateau. Thus, we choose the following general relationship between fecundity and average gain:

$$
F\left(\bar{G}_{i}\right)=F \frac{\bar{G}_{i}}{\gamma+\bar{G}_{i}} \text { where } i \in\{H, D\} .
$$


We use the average gains of Hawk and Dove of Eqs. (5) and (6) to calculate their respective fecundities. Fecundity is an increasing function of the average gain. Parameter $\gamma$ permits to influence the "speed" to reach the plateau of the fecundity. When $\gamma$ decreases the maximum of the fecundity is reached more quickly.

\subsection{The complete slow-fast model}

The complete model is obtained by coupling the previous hawk-dove behavioral model and the populations model as follows:

$$
\left\{\begin{array}{l}
\varepsilon \frac{d n_{1}}{d t}=\varepsilon\left(F\left(\frac{\overline{G_{H}}}{\gamma+\overline{G_{H}}} n_{2}^{H}+\frac{\overline{G_{D}}}{\gamma+\bar{G}_{D}} n_{2}^{D}\right)-\left(\mu_{1}+\beta\right) n_{1}-\eta n_{1}^{2}\right) \\
\varepsilon \frac{d n_{2}^{H}}{d t}=n_{2} x\left(\Delta_{H}-\Delta\right)+\varepsilon\left(q \beta n_{1}-\left(\mu+\alpha \frac{C}{2} \frac{n_{2}^{H}}{n_{2}}\right) n_{2}^{H}\right) \\
\varepsilon \frac{d n_{2}^{D}}{d t}=n_{2} y\left(\Delta_{D}-\Delta\right)+\varepsilon\left((1-q) \beta n_{1}-\mu n_{2}^{D}\right)
\end{array}\right.
$$

where $\varepsilon$ is a small parameter. It is also usual to use the fast time scale $\tau$

$$
\left\{\begin{array}{l}
\frac{d n_{1}}{d \tau}=\varepsilon\left(F\left(\frac{\overline{G_{H}}}{\gamma+\overline{G_{H}}} n_{2}^{H}+\frac{\overline{G_{D}}}{\gamma+\overline{G_{D}}} n_{2}^{D}\right)-\left(\mu_{1}+\beta\right) n_{1}-\eta n_{1}^{2}\right) \\
\frac{d n_{2}^{H}}{d \tau}=n_{2} x\left(\Delta_{H}-\Delta\right)+\varepsilon\left(q \beta n_{1}-\left(\mu+\alpha \frac{C}{2} \frac{n_{2}^{H}}{n_{2}}\right) n_{2}^{H}\right) \\
\frac{d n_{2}^{D}}{d \tau}=n_{2} y\left(\Delta_{D}-\Delta\right)+\varepsilon\left((1-q) \beta n_{1}-\mu n_{2}^{D}\right)
\end{array}\right.
$$

In this last form, it is obvious that the game dynamics correspond to the fast time scale while the small terms of the order of $\varepsilon$ correspond to the slow time scale. This model is a three-dimensional system of ordinary differential equations.

\section{The aggregated model}

When parameter $\varepsilon$ is small (see Eq. (8)), two different time scales are involved in the dynamics, a fast one corresponding to the game and a slow one corresponding to the demographic model. We shall now take advantage of the two time scales to reduce the dimension of the complete system (8) of three equations into a system of two equations. For aggregation methods, we refer to Iwasa et al., 1987 and 1989; Auger and Bravo de la Parra, 2000; Auger et al. 2008a and 2008b; and Sanz and Bravo de la Parra, 1999. In the next step, we shall proceed to the analysis of this 'aggregated' model which will be simpler to study than the complete one. 


\subsection{Derivation of the aggregated model}

The first step is to neglect the small terms of the order of $\varepsilon$ and to look for the existence of a stable equilibrium for the fast part of the system which relates to the game dynamics

$$
\left\{\begin{array}{l}
\frac{d x}{d \tau}=x\left(\Delta_{H}-\Delta\right) \\
\frac{d y}{d \tau}=y\left(\Delta_{D}-\Delta\right)
\end{array}\right.
$$

Using the fact that $x+y=1$ at any time $t$ and after some algebra, the previous system can be reduced to a single equation governing the hawk proportion of individual. In case of constant gains and costs, it reads

$$
\frac{d x}{d t}=\frac{x}{2}(1-x)(G-C x) .
$$

This equation has three equilibria, 0,1 and $\frac{G}{C}$. 0 is always unstable. Let us denote $x^{*}$ the stable non-trivial equilibrium. According to parameters values, two cases can occur:

- $G<C, x^{*}=\frac{G}{C}$ is asymptotically stable for any initial condition $0<x(0)<1$. In this case, at equilibrium, the population is polymorphic with a proportion $\frac{G}{C}$ of hawks and $1-\frac{G}{C}$ of doves.

When replacing the variables $n_{2}^{H}$ and $n_{2}^{D}$ by those of the fast equilibrium, the average costs and gains become:

$$
\begin{array}{ll}
\overline{C_{H}}=\frac{G}{2} & \overline{C_{D}}=0 \\
\overline{G_{H}}=G\left(1-\frac{G}{2 C}\right) & \overline{G_{D}}=\frac{G}{2}\left(1-\frac{G}{C}\right)
\end{array}
$$

- $G>C, x^{*}=\frac{G}{C}$ does not belong to the interval $[0,1]$. The equilibrium $x^{*}=1$ is asymptotically stable. The population is monomorphic and totally hawk at equilibrium.

When replacing the variables $n_{2}^{H}$ and $n_{2}^{D}$ of the complete model by those of the fast equilibrium, the average costs and gains become:

$$
\begin{array}{ll}
\overline{C_{H}}=\frac{C}{2} & \overline{C_{D}}=0 \\
\overline{G_{H}}=\frac{G}{2} & \overline{G_{D}}=0
\end{array}
$$

In order to aggregate, we make the assumption that the fast process is at the fast equilibrium. Thus, we come back to the complete initial system (8), substitute the previous fast equilibrium and add the two matures equations. It is necessary to replace the fast variables in terms of the fast equilibrium as follows:

$$
n_{2}^{H}=x^{*} n_{2} \text { and } n_{2}^{D}=\left(1-x^{*}\right) n_{2}
$$


After some algebra, one obtains the following system of two equations governing the immature and mature densities at the slow time scale, that we call the aggregated model

$$
\left\{\begin{array}{l}
\frac{d n_{1}}{d t}=F\left(\frac{\overline{G_{H}}}{\gamma+G_{H}} x^{*}+\frac{\overline{G_{D}}}{\gamma+G_{D}}\left(1-x^{*}\right)\right) n_{2}-\left(\mu_{1}+\beta\right) n_{1}-\eta n_{1}^{2} \\
\frac{d n_{2}}{d t}=\beta n_{1}-\mu n_{2}-\alpha \frac{C}{2}\left(x^{*}\right)^{2} n_{2}
\end{array}\right.
$$

The dynamics of the aggregated system (10) is a good approximation of the dynamics of the complete system (8) provided the aggregated system is structurally stable, which is the case (see section 3.2 for the analysis); and $\varepsilon$ is small enough, which is assumed. The more detail about aggregation method can be seen in Auger et al. 2008 a and 2008b.

In our case, we remember that we can have two possibilities for the fast equilibrium, then we obtain two different aggregated models which are valid on two domains of the phase plane:

- Model I: $G<C, x^{*}=\frac{G}{C}$ is asymptotically stable in Eq. (9),

$$
\left\{\begin{array}{l}
\frac{d n_{1}}{d t}=F\left(\frac{\overline{G_{H}}}{\gamma+\overline{G_{H}}} \frac{G}{C}+\frac{\overline{G_{D}}}{\gamma+\overline{G_{D}}}\left(1-\frac{G}{C}\right)\right) n_{2}-\left(\mu_{1}+\beta\right) n_{1}-\eta n_{1}^{2} \\
\frac{d n_{2}}{d t}=\beta n_{1}-\mu n_{2}-\alpha \frac{G^{2}}{2 C} n_{2}
\end{array}\right.
$$

where

$$
\bar{G}_{H}=G\left(1-\frac{G}{2 C}\right), \quad{\overline{G_{D}}}=\frac{G}{2}\left(1-\frac{G}{C}\right)
$$

- Model II: $G>C, x^{*}=1$ is asymptotically stable in Eq. (9),

$$
\left\{\begin{array}{l}
\frac{d n_{1}}{d t}=F \frac{\overline{G_{H}}}{\gamma+\bar{G}_{H}} n_{2}-\left(\mu_{1}+\beta\right) n_{1}-\eta n_{1}^{2} \\
\frac{d n_{2}}{d t}=\beta n_{1}-\mu n_{2}-\alpha \frac{C}{2} n_{2}
\end{array}\right.
$$

where $\overline{G_{H}}=\frac{G}{2}$.

It must be noted that there is continuity, i.e. average gains and costs in mixed and pure hawk cases become equal at the separation line between the two models, when $G=C$, in this case, these two models connect.

\subsection{Study of the dynamics of the aggregated model}

We set up the stage structured model which general form

$$
\left\{\begin{array}{l}
\frac{d n_{1}}{d t}=F_{1} n_{2}-\left(\mu_{1}+\beta\right) n_{1}-\eta n_{1}^{2}=P\left(n_{1}, n_{2}\right) \\
\frac{d n_{2}}{d t}=\beta n_{1}-r n_{2}=Q\left(n_{1}, n_{2}\right)
\end{array}\right.
$$

where 
$F_{1}=F\left(\frac{\overline{G_{H}}}{\gamma+\overline{G_{H}}} \frac{G}{C}+\frac{\overline{G_{D}}}{\gamma+\overline{G_{D}}}\left(1-\frac{G}{C}\right)\right), r=\mu+\alpha \frac{G^{2}}{2 C}$ in the case of mixed strategy and

$F_{1}=F \frac{\overline{G_{H}}}{\gamma+\overline{G_{H}}}, r=\mu+\alpha \frac{C}{2}$ in the case of pure hawk population.

The initial conditions for system (13) take the form of

$$
n_{1}(0)>0, n_{2}(0)>0 \text {. }
$$

Considering the biological significance, we study system (13) in the region

$$
D=\left\{\left(n_{1}, n_{2}\right) \in R^{2}: n_{1} \geq 0, n_{2} \geq 0\right\} .
$$

Hence, the following assertion is of major importance.

Lemma 1 The non-negative quadrant of $\mathbb{R}^{2}$ is positively invariant for system (13).

Proof 1 Let $X=\left(n_{1}, n_{2}\right)^{T}$. For the system (13), we notice that

$$
\begin{aligned}
& \left.P\right|_{n_{1}=0, X \in \mathbb{R}_{+}^{2}}=F_{1} n_{2}-\left(\mu_{1}+\beta\right) n_{1}-\eta n_{1}^{2}=F_{1} n_{2} \geq 0, \\
& \left.Q\right|_{n_{2}=0, X \in \mathbb{R}_{+}^{2}}=\beta n_{1}-r n_{2}=\beta n_{1} \geq 0 .
\end{aligned}
$$

Then $\mathbb{R}_{+}^{2}$ is an invariant domain of (13).

of

Now we carry out existence and stability of the equilibria of (13), which are solution

$$
\left\{\begin{array}{l}
F_{1} n_{2}-\left(\mu_{1}+\beta\right) n_{1}-\eta n_{1}^{2}=0 \\
\beta n_{1}-r n_{2}=0
\end{array}\right.
$$

We are only interested in the non-negative equilibria.

\section{Proposition. 1}

$(0,0)$ is always an equilibrium of system $(13)$.

1. If $F_{1} \beta<\left(\mu_{1}+\beta\right) r$, then $(0,0)$ is a stable node.

2. If $F_{1} \beta>\left(\mu_{1}+\beta\right) r$, then $(0,0)$ is a saddle point.

\section{Proof}

Obviously, $(0,0)$ is an equilibrium system (13). The Jacobian matrix corresponding to the linearized system of (13) is

$$
J\left(n_{1}, n_{2}\right)=\left(\begin{array}{cc}
-\mu_{1}-\beta-2 \eta n_{1} & F_{1} \\
\beta & -r
\end{array}\right) .
$$

For the equilibrium $(0,0)$, Jacobian is reduces to

$$
J_{0}=\left(\begin{array}{cc}
-\mu_{1}-\beta & F_{1} \\
\beta & -r
\end{array}\right) .
$$


Hence, the stability of $(0,0)$ determined by the characteristic equation's eigenvalues

$$
\left(\lambda+\mu_{1}+\beta\right)(\lambda+r)-F_{1} \beta=0 .
$$

Solving it produces

$$
\lambda_{1}+\lambda_{2}=-\left(\mu_{1}+\beta+r\right)
$$

and

$$
\lambda_{1} \lambda_{2}=r\left(\mu_{1}+\beta\right)-F_{1} \beta .
$$

We have

$$
\begin{aligned}
\Delta & =\left(\mu_{1}+\beta+r\right)^{2}-4 r\left(\mu_{1}+\beta\right)+4 F_{1} \beta \\
& =\left(\mu_{1}+\beta-r\right)^{2}+4 F_{1} \beta>0 .
\end{aligned}
$$

Hence, when (1) is satisfied, $(0,0)$ is a stable node. When (2) is satisfied, $(0,0)$ is a saddle point. This completes the proof.

The positive equilibrium of system (13) is the intersection of the isoclines

$$
\left\{\begin{array}{l}
F_{1} n_{2}-\left(\mu_{1}+\beta\right) n_{1}-\eta n_{1}^{2}=0, \\
\beta n_{1}-r n_{2}=0
\end{array}\right.
$$

By simple calculation we have

$$
\begin{aligned}
& n_{1}^{*}=\frac{F_{1} \beta-r\left(\mu_{1}+\beta\right)}{r \eta}, \\
& n_{2}^{*}=\frac{\beta}{r} n_{1}^{*} .
\end{aligned}
$$

To ensure the positivity of the equilibrium $\left(n_{1}^{*}, n_{2}^{*}\right)$, we assume that

$$
F_{1} \beta>r\left(\mu_{1}+\beta\right)
$$

\section{Proposition. 2}

If $F_{1} \beta>r\left(\mu_{1}+\beta\right)$, then there exists a unique positive equilibrium $\left(n_{1}^{*}, n_{2}^{*}\right)$, which is a stable node. If $F_{1} \beta<r\left(\mu_{1}+\beta\right)$, then there exists no positive equilibrium, $(0,0)$ is the unique equilibrium in the positive quadrant $D$.

\section{Proof}

From the above discussion, the existence of the positive equilibrium is over. Now, we analyse the local geometric properties of $\left(n_{1}^{*}, n_{2}^{*}\right)$. The Jacobian matrix of $\left(n_{1}^{*}, n_{2}^{*}\right)$ is

$$
J\left(n_{1}^{*}, n_{2}^{*}\right)=\left(\begin{array}{cc}
-\mu_{1}-\beta-2 \eta n_{1}^{*} & F_{1} \\
\beta & -r
\end{array}\right) .
$$


For the Jacobian also can be reduced to

$$
J\left(n_{1}^{*}, n_{2}^{*}\right)=\left(\begin{array}{cc}
-F_{1} \frac{n_{2}^{*}}{n_{1}^{*}}-\eta n_{1}^{*} & F_{1} \\
\beta & -r
\end{array}\right) .
$$

Hence, the stability of $\left(n_{1}^{*}, n_{2}^{*}\right)$ determined by the characteristic equation's eigenvalues

$$
\lambda^{2}+\left(F \frac{n_{2}^{*}}{n_{1}^{*}}+\eta n_{1}^{*}+r\right) \lambda+r\left(F \frac{n_{2}^{*}}{n_{1}^{*}}+\eta n_{1}^{*}\right)-F_{1} \beta
$$

It is not hard to see that when $F_{1} \beta>r\left(\mu_{1}+\beta\right),(16)$ has two negative roots, thus $\left(n_{1}^{*}, n_{2}^{*}\right)$ is a stable node. The proof is completed.

\section{Proposition. 3}

If $F_{1} \beta>r\left(\mu_{1}+\beta\right)$, then the unique positive equilibrium $\left(n_{1}^{*}, n_{2}^{*}\right)$ is globally asymptotically stable.

\section{Proof}

It is easy to see that in $D, \frac{\partial P}{\partial n_{1}}+\frac{\partial Q}{\partial n_{2}}=-\mu_{1}-\beta-2 \eta n_{1}-r<0$ then By PoincareBendixson theorem, there are no limit cycles in $D$, and $\left(n_{1}^{*}, n_{2}^{*}\right)$ is the unique positive equilibrium which is stable node in $D$, so it is globally asymptotically stable. This completes the proof.

\section{Proposition. 4}

If $F_{1} \beta<r\left(\mu_{1}+\beta\right)$, then the unique non negative equilibrium $(0,0)$ is globally asymptotically stable.

\section{Proof}

We construct the following Liapunov function

$$
V\left(n_{1}, n_{2}\right)=\beta n_{1}+\left(\mu_{1}+\beta\right) n_{2} .
$$

Calculating the derivative of $V\left(n_{1}, n_{2}\right)$ along (13), we have

$$
\frac{d V}{d t}\left(n_{1}, n_{2}\right)=\left(F_{1} \beta-r\left(\mu_{1}+\beta\right)\right) n_{2}-\beta \eta n_{1}^{2} \leq 0 .
$$

We can see that in the domain $D, \frac{d V}{d t}=0$ if and only if $n_{1}=0, n_{2}=0$. Hence $(0,0)$ is globally asymptotically stable. This completes the proof.

\section{Discussion and Conclusion}

We have presented a stage structured population system with mature individuals using hawk and dove tactics. Our model is based on the coupling between population dynamics 


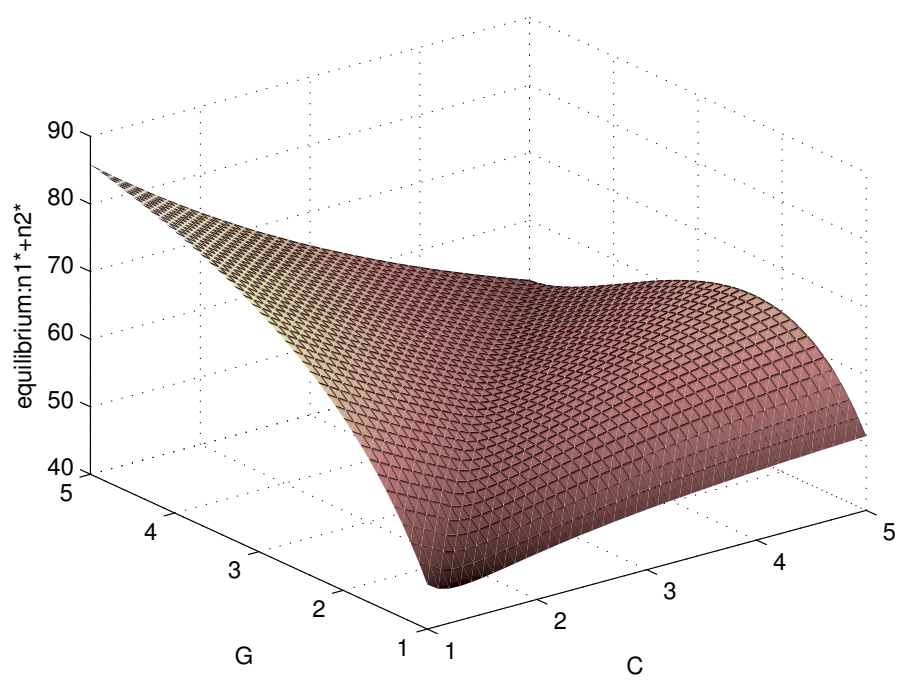

Figure 1. Total density of the population as a function of gain (G) and cost (C).

and game dynamics. This coupling allowed us to describe the overall population dynamics, with individuals using different tactics during their life and to analyse the effects of different behavioral changes on population growth according to biological processes going on at different time scales (day for contests, year for the demography).

Aggregation methods have been applied to the study of dynamical systems with two time scales (Auger and Poggiale, 1998; Iwasa et al.,1987 and 1989; Auger and Bravo de la Parra, 2000; Auger et al. 2008a and 2008b; Auger and Poggiale, 1998; Sanchez et al. 1995 ). In our model, the game operates on a fast-time scale and the demography on a slow-time scale. Assuming that the fast process goes to a stable equilibrium, the aggregated model can be used to evaluate the consequences of changing the proportion of hawks and to study the consequences of changes in individual behavior on population equilibrium.

As a first result, figure 1 shows the total density of the population, immature and mature, with respect to gain and cost. Regarding game parameters, $C$-values can be considered as a gradient of aggressiveness, from non aggressive species (small $C$ ) to very aggressive, (very large $C$ ). $G$-values can represent a gradient of environment, from poor with few resource (small $G$ ) to rich environment (large $G$ ). Figure 1 shows the graph of the total density of the population with respect to gain and cost with two different domains : " A domain $G<C$ in which the population is mixed with a proportion of hawks $x^{*}=\frac{G}{C}$ at the fast equilibrium. " A domain $G>C$ in which the population 
is pure hawk at the fast equilibrium. In the domain $(G<C)$, there is a kind of bump (figure 1) with a curved line corresponding to maximum fitness. In other words, for each fixed $\operatorname{cost} C$, there is a maximum gain. Each point along this curved line of maxima with coordinates $(G, C)$ corresponds to a given proportion of hawks which is simply given by $x^{*}=G / C$. The total density of the population is maximized when the proportion of aggressive individuals is smaller for larger cost. When the cost is high, according to this model, we expect a population mainly dove with few hawks. On the contrary, when the cost is small, we expect a larger proportion of hawks in the population but with small costs, corresponding to less injuries leading to smaller mortality. In the domain $(G>C)$, the graph of the fitness is monotone increasing with $G$ for any cost-values. The result shows that an increase in resource value may decrease total population density, because it promotes individual (i.e. selfish) behavior. Our results may therefore find practical implications in animal conservation or biological control for instance.

\section{Acknowledgements}

The authors would like to thank the editor and anonymous referees for their careful reading of the manuscript and valuable suggestions to improve the quality of this work. This work was supported by the National Research Project: CNEPRU B02020140100, Tassili Project 10MDU800 and by Vietnam National Foundation for Science and Technology Development (NAFOSTED) under grant number 101.02-2013.18. A part of this work was done while Ali Moussaoui was at Applied Mathematics Laboratory of Le Havre as Research Associate (FR CNRS 3335) and while Doanh Nguyen-Ngoc was at Vietnam Institute for Advanced Study in Mathematics (VIASM), Hanoi, Vietnam.

\section{References}

Auger P., Bravo de la Parra R., Sanchez, E., 1998. Hawk-dove games and competition dynamics, Math. Comp. Mod. 27 89-98.

Auger P., Bravo de la Parra R., 2000. Methods of aggregation of variables in population dynamics. C. R. Sciences de la Vie, 323, 665-674.

Auger P., Bravo de la Parra R., Sanchez E., 2001. Behavioral Dynamics of Two Interacting HawkDove Populations, Mathematical Models and Methods in Applied Sciences (M3AS), 11(4), pp. 645-661.

Auger P., Bravo de la Parra R., Morand S., Sanchez E., 2002. A predator-prey model with predators using hawk and dove tactics. Math. Biosci. 177-178, 185-200.

Auger P., Bravo de la Parra R., Poggicale J.C., Sanchez E., Nguyen-Huu T., 2008a. Aggregation of Variables and Applications to Population Dynamics, in Magal P, Ruan S (eds.), Structured Population Models in Biology and Epidemiology, Lecture Notes in Mathematics, Vol 1936, 
Mathematical Biosciences Subseries, Springer, Berlin, pp. 209-263.

Auger P., Bravo de la Parra R., Poggiale J.C., Sanchez E., Sanz L., 2008b. Aggregation methods in dynamical systems variables and applications in population and community dynamics, Physics of Life Reviews 5: 79-105.

Auger P., Pontier D., 1998., Fast game theory coupled to slow population dynamics: The case of domestic cat populations, Math. Biosci. 148. 65-82.

Auger P., Kooi B.W., Bravo de la Parra R., Poggiale J.C., 2006. Bifurcation analysis of a predator-prey model with predators using hawk and dove tactics, Journal of Theoretical Biology, 238(3):587-607.

Auger P., Poggiale J.C, 1998. Aggregation and Emergence in Systems of Ordinary Differential Equations, Mathematical Computer Modelling, 27, 4, pp. 1-22.

Begon M., Mortimer, M., Thompson D. J., 1996. Population ecology: a unified study of animals and plants, 3rd edn. Blackwell Science, Malden, MA. 247 pp.

Chambon-Dubreuil. E., Auger P., Gaillard J.M., Khaladi M.,2006. Effect of aggressive behaviour on age-structured population dynamics. Ecological Modelling. 193, 777-786.

Cressman. R, 1992. The Stability Concept of Evolutionary Game Theory, Lecture Notes in Biomathematics, Vol. 94. Springer.

Cressman. R, 1995. Evolutionary game theory with two groups of individuals, Games Econ. Behavior 11. 237-253.

Hofbauer J., Sigmund. K., 1998. Evolutionary Games and Population Dynamics. Cambridge Univ. Press.

Iwasa Y., Endreasen V., Levin S. A., 1987, Aggregation in model ecosystems I. Perfect aggregation, Ecol. Modell. 37, 287.

Iwasa Y., Endreasen V., Levin S. A., 1989. Aggregation in model ecosystems ii. approximate aggregation. IMA J. Math. Appl. Med. Biol, 6, 1-23.

Davies N.B., Kreb J.R., 1993. An Introduction to Behavioural Ecology. Wiley-Blackwell, 3rd Edition edition, 432 pages.

Lott D.F., 1991. Intraspecific Variation in the Social Systems of Wild Vertebrates. Cambridge University, New York.

Manning A., Dawkins M.S., 1998. An Introduction to Animal Behaviour. Cambridge University Press, 450 pages.

Marva M, Moussaoui A, Bravo de la Parra R., Auger P., 2013. A density-dependent model describing age-structured population dynamics using hawk-dove tactics. Journal of Difference Equations and Applications. Vol. 19, No. 6, June 2013, 1022-1034.

Mesterton-Gibbons M., 1992. An Introduction to Game-Theoretic Modell., Addison-Wesley, Redwood City.

Maynard Smith J., 1982. Evolution and Theory of Games (Cambridge Univ. Press).

Moussaoui A., Auger P., Roche B., 2014. Effect of Hawk-Dove Game on the Dynamics of Two Competing Species. Acta Biotheor 62:385-404. 
Moussaoui A., Nguyen-Ngoc D., Auger P., 2012. A stage structured population model with mature individuals using hawk and dove tactics. 11th African Conference on Research in Computer Science and Applied Mathematics, CARI'2012. Algiers, Algeria, 251-258.

Sanz L., Bravo de la Parra R., 1999. Variables aggregation in a time discrete linear model. Mathematical Biosciences 157, 111-146.

Sanchez E., Bravo de la Parra R., Auger P., 1995. Linear Discrete Models with Different Time Scales. Acta Biotheoretica 43 (4) 465-479.

Sigmund K., 1986. A survey of replicator equations. Complexity, Language, and Life: Mathematical Approaches, Springer Berlin Heidelberg, 88-104.

Sutherland W. J., Norris K., 2002. Behavioural models of population growth rates: implications for conservation and prediction. Phil. Trans. R. Soc. Lond. B 357, 1273-1284. 